\title{
Resultados de sobrevida no médio prazo da haste femoral não cimentada Logical*
}

\section{Results of Medium-Term Survival of the Logical Non-Cemented Logical Femoral Stem}

\author{
Cristiano Valter Diesel1,2,30 Tiango Aguiar Ribeiro ${ }^{1,4,5}$ Carlos Alberto de Souza Macedo ${ }^{2,3}$ \\ Carlos Roberto Galia ${ }^{1,2,3}$
}

1 Programa de Pós-Graduação em Medicina, Ciências Cirúrgicas, Universidade Federal do Rio Grande do Sul (UFRGS), Porto Alegre, RS, Brasil

${ }^{2}$ Serviço de Ortopedia e Traumatologia, Hospital de Clínicas de Porto Alegre (HCPA), Porto Alegre, RS, Brasil

3 Departamento de Cirurgia, Faculdade de Medicina (FAMED), Universidade Federal do Rio Grande do Sul (UFRGS), Porto Alegre, RS, Brasil

4 Departamento de Cirurgia, Curso de Medicina, Universidade Federal de Santa Maria (UFSM), Santa Maria, RS, Brasil

${ }^{5}$ Serviço de Ortopedia e Traumatologia, Hospital Universitário de

Santa Maria (SOT-HUSM), Santa Maria, RS, Brasil

\begin{abstract}
Endereço para correspondência Cristiano Valter Diesel, MD, MSc, Serviço de Ortopedia e Traumatologia do Hospital de Clínicas de Porto Alegre, Rua Ramiro Barcelos, 2350, Porto Alegre, Rio Grande do Sul, Brasil. CEP: 90035-903

(e-mail: cristianodiesel@gmail.com; cristianodiesel@ymail.com).
\end{abstract}

\section{Resumo}

Palavras-chave

- artroplastia de quadril

- cimentos para ossos

- articulação do quadril

- estudos de acompanhamento
Objetivo Avaliar os resultados clínicos e radiográficos da haste femoral não cimentada Logical (Baumer, Mogi Mirim, SP, Brasil) nas artroplastias totais do quadril (ATQs). Método Foi realizada uma coorte retrospectiva de 632 pacientes submetidos à artroplastia primária do quadril por coxartrose nos quais foram implantadas hastes femorais não cimentadas Logical. Todos os pacientes eram provenientes do ambulatório de Cirurgia do Quadril da instituição. O período do estudo compreendeu de janeiro de 2004 a janeiro de 2015. O desfecho definido para avaliar a sobrevida da haste foi a indicação clínica e radiográfica da revisão ou a revisão propriamente dita da haste femoral por qualquer causa.

Resultado Observaram-se curvas de sobrevida estimadas $>95 \%$, com um seguimento variando de 2 até 13 anos. Houve baixa incidência de fraturas periprotéticas transoperatórias $(0,02 \%)$. Não foi observada migração axial ou atrofia óssea cortical na amostra radiográfica avaliada.

Conclusão Os resultados demonstram, no médio prazo, excelente sobrevida da haste femoral não cimentada Logical. Embora ainda sejam aguardados estudos no longo prazo, este implante parece ser seguro e promissor para ser utilizado nas artroplastias primárias do quadril.

* Trabalho feito no Hospital de Clínicas de Porto Alegre (HCPA); Serviço de Ortopedia e Traumatologia; Ambulatório de Cirurgia do Quadril, Porto Alegre, RS, Brasil.

(D) Cristiano Valter Diesel's ORCID is https://orcid.org/0000-00015295-9852.

recebido

31 de Março de 2018

aceito

10 de Julho de 2018
DOI https://doi.org/

10.1055/s-0039-1688755. ISSN 0102-3616.
Copyright $\odot 2019$ by Sociedade Brasileira License terms de Ortopedia e Traumatologia. Published by Thieme Revnter Publicações Ltda, Rio de Janeiro, Brazil 


\author{
Abstract \\ Keywords \\ - arthroplasty, \\ replacement, hip \\ - bone cements \\ - hip joint \\ - follow-up studies
}

Objective The main objective of the present study was to evaluate the clinical and radiographic results of the Logical (Baumer, Mogi Mirim, SP, Brasil) cementless femoral stem in primary total hip arthroplasties (THAs).

Methods A retrospective cohort study of 632 patients submitted to primary THA with Logical cementless femoral stem. The study period was between January 2004 and January 2015. The outcome defined to evaluate the survival of the stem was the clinical and radiographic indication of the revision hip arthroplasty or the actual revision of the femoral stem for any cause.

Results Kaplan-Meier survival curves were estimated $>95 \%$, with a follow-up ranging from 2 to 13 years. There was a low incidence of transoperative periprosthetic fractures $(0.02 \%)$. No axial migration or cortical bone atrophy was observed in the radiographic sample evaluated.

Conclusions In the intermediate follow-up, there was excellent survival of the Logical cementless femoral stem. Although long-term studies are still awaited, this implant appears to be safe and promising to be used for primary THAs.

\section{Introdução}

As hastes não cimentadas são amplamente utilizadas nas artroplastias total de quadril (ATQs). No entanto, o termo não cimentado é uma descrição genérica e denomina um grande grupo de implantes que não utiliza o polimetilmetacrilato (PMNA) como interface entre o osso hospedeiro e a prótese. A inexistência do PMNA exige que a haste tenha uma fixação direta no osso, sendo assim, mais dependente da forma, da porosidade, do tipo de metal empregado e do design.

Existe grande diversidade na combinação dessas características, fato que, por fim, gera resultados variáveis dos implantes não cimentados. Diversos modelos de hastes apresentaram excelentes desfechos clínicos e radiográficos; outros, porém, não tiveram este bom desempenho, e alguns demonstraram resultados catastróficos. ${ }^{1-3}$

Nos anos 1990, embora o mundo apresentasse um boom nas hastes não cimentadas e uma facilidade no acesso a estes implantes, alguns países, por peculiaridades socioeconômicas, experimentaram escassez e dificuldade de acesso aos implantes não cimentados de qualidade. Esta, a nosso ver, foi a situação do Brasil ou de grande parte do país.

Neste cenário, iniciou-se o projeto de desenvolvimento de uma haste não cimentada com design quadrangular e dupla cunha, considerado pelos autores o mais adequado. Além disso, a haste deveria ter todos os testes exigidos pela Administração de Comidas e Remédios dos Estados Unidos (FDA, na sigla em inglês), pela American Society for Testing and Materials (ASTM) e, sobretudo, alto padrão de qualidade quando submetida aos mais rigorosos testes laboratoriais.

O resultado foi a produção de um implante não cimentado, com núcleo básico reto quadrangular, em dupla cunha, com aletas nas faces anterior e posterior, forjada em uma liga de titânio, alumínio e vanádio. 0 ângulo cérvico-diafisário adotado foi de $135^{\circ}$, e com cone Morse $12 / 14 \mathrm{~mm}$. Todo o implante é recoberto por jateamento com textura grossa e seu terço proximal recebe uma camada porosa circunferencial de titânio puro depositado por termo aspersão (plasma spray). Estes implantes foram produzidos nos tamanhos $8,9,10,11,25$, $12,5,13,75$ e 15 . Hastes mais robustas, de tamanhos 16,18 e 20 , possuem características similares, porém a camada de titânio recobre quase a totalidade da haste $\mathbf{4}^{4}$ ( Fig. 1).

Apesar dos primeiros passos serem exitosos e as premissas básicas em termos de metalurgia, dos rigorosos testes biomecânicos, dos testes com elementos finitos e o controle laboratorial validarem a produção e a qualidade do implante, ${ }^{4}$ ainda não atestam e nem garantem o seu sucesso no ambiente clínico, ou seja, quando submetida a toda a sorte de situações in vivo (-Fig. 2).

Com o objetivo de avaliar o desempenho da haste não cimentada Logical (Baumer, Mogi Mirim, SP, Brasil), realizamos uma avaliação de todos os casos de artroplastia primária do quadril que utilizaram este implante em nossa instituição, entre os anos de 2004 e 2015. Preencheram os critérios de inclusão 632 pacientes.

\section{Material e Métodos}

Todos os pacientes que foram submetidos a ATQ com haste Logical, entre os anos de 2004 e 2015, seguidos no ambulatório de Cirurgia do Quadril, tiveram seus dados coletados no sistema de arquivo eletrônico e físico. Embora o banco de dados contivesse $>1.000$ pacientes, foram excluídos aqueles nos quais a haste foi utilizada nas fraturas do fêmur proximal, nas revisões de artroplastia e nas fraturas decorrentes de metástases ósseas ou de tumores ósseos primários. Restaram 632 pacientes.

Todas as cirurgias foram realizadas ou supervisionadas por quatro ortopedistas especialistas em cirurgia do quadril. A via posterolateral foi utilizada em todos os casos. A profilaxia com antibiótico adotada foi a cefazolina endovenosa conforme o protocolo institucional e a tromboprofilaxia pós-operatória foi realizada com enoxaparina subcutânea. Os pacientes retornaram no $15^{\circ}, 45^{\circ}$ e $90^{\circ}$ dias do pósoperatório; após este período inicial, os retornos ocorriam a cada 6 meses nos primeiros 18 meses e então anualmente. 

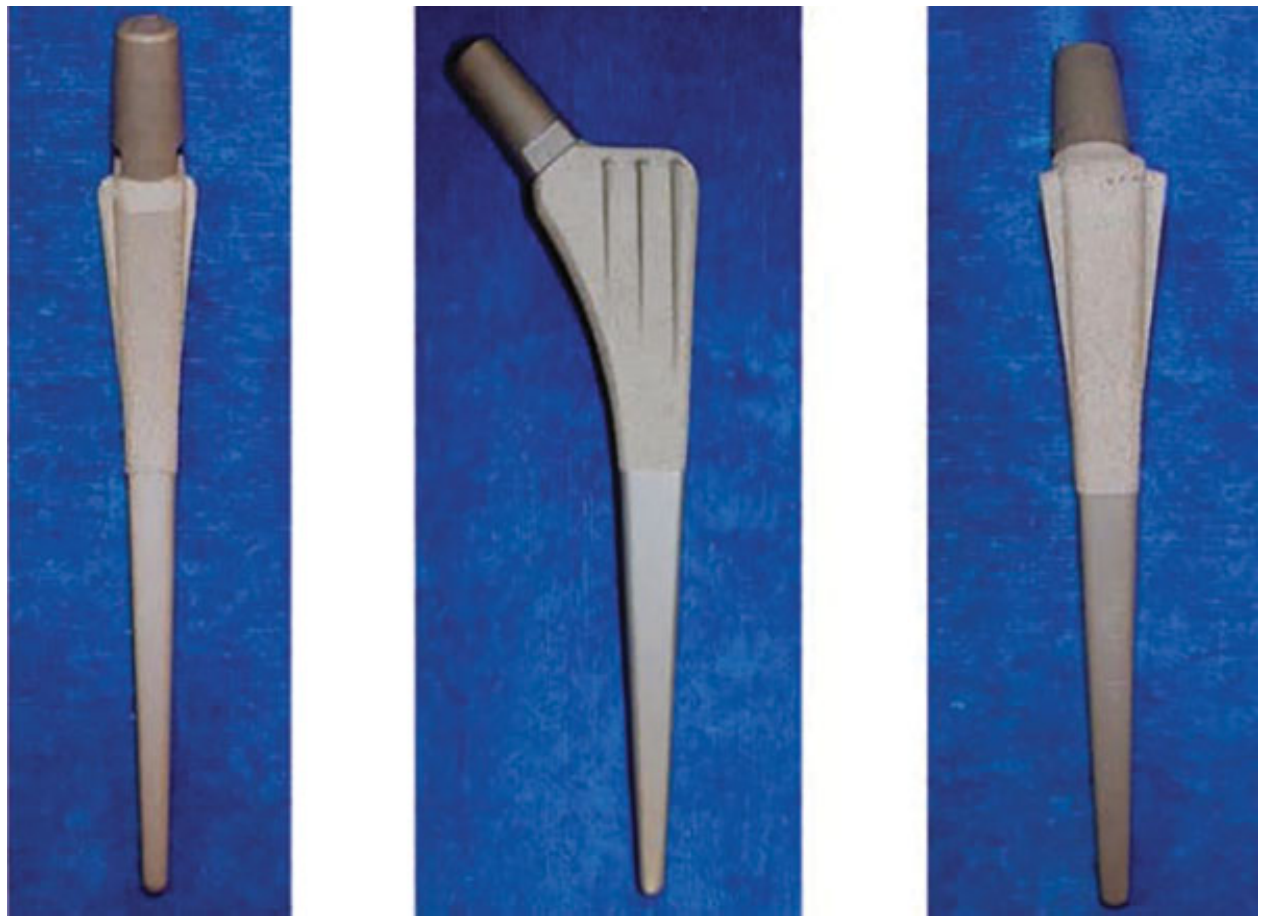

Fig. 1 Haste femoral não cimentada Logical - modelo inicial. ${ }^{4}$
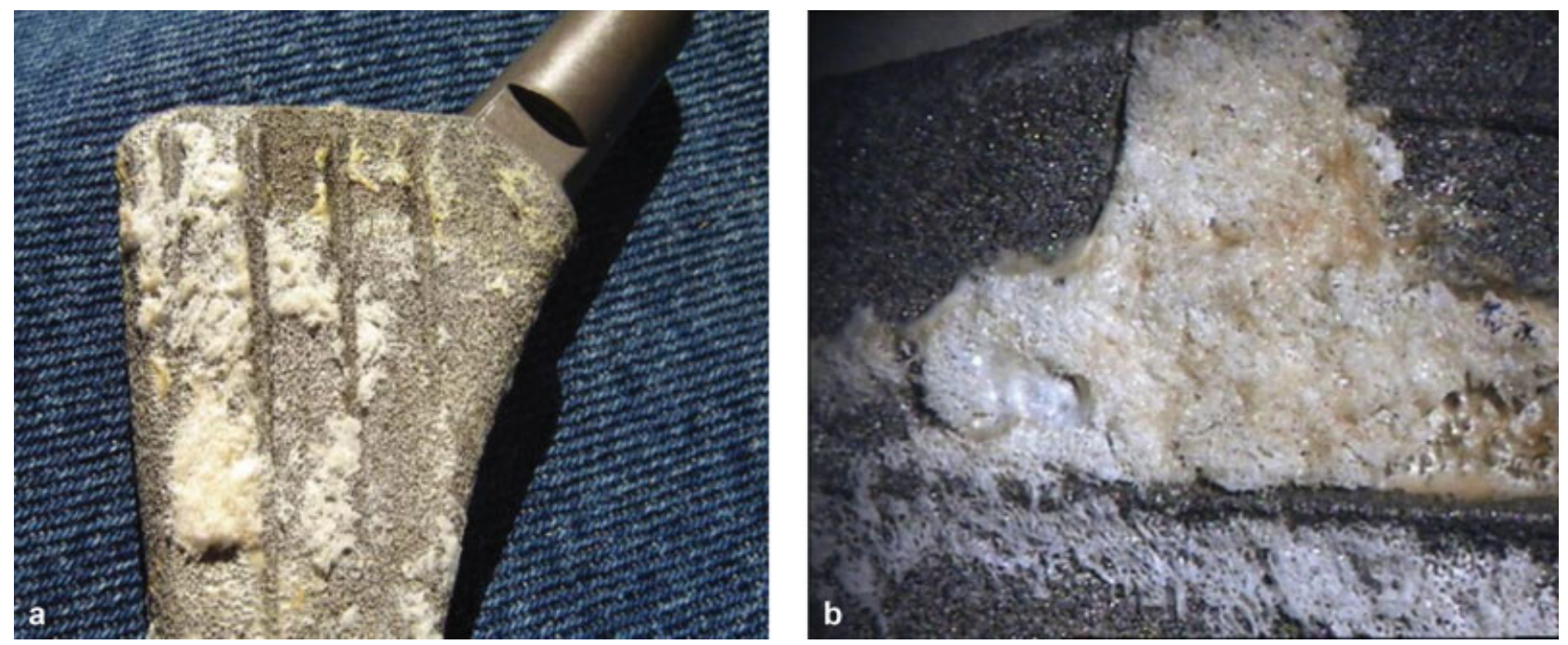

Fig. 2 Fotografias de uma haste Logical removida devido a infecção. Imagens demostrando nítido crescimento ósseo para a superfície porosa da haste.

Radiografias de controle foram obtidas no pós-operatório imediato, no $45^{\circ}$ dia, e no $180^{\circ}$ dia. A partir disso, os exames radiográficos eram realizados anualmente.

O desfecho avaliado foi a necessidade de revisão do componente femoral por sinais radiográficos de afrouxamento do componente femoral ou por dor associada à cirurgia. Para fins de desfecho, consideramos a data da indicação cirúrgica como a data da falha do implante.

Foram adotados os seguintes critérios de inclusão: ter realizado ATQ primária por artrose do quadril, primária ou secundária, com a haste Logical, e ter realizado seguimento periódico no serviço de ortopedia. Foram excluídos os pacientes submetidos a artroplastia parcial ou bipolar do quadril por fratura do fêmur proximal, as revisões de artro- plastia com haste Logical, e os pacientes operados devido a fratura patológica do fêmur proximal.

Tabularam-se os dados epidemiológicos como gênero, idade e indicação cirúrgica. Também foi coletado o tamanho dos implantes utilizados, além dos índices de infecção, de fratura femoral transoperatória e de fratura periprotética pós-operatória.

Devido ao grande número de casos, foi selecionada aleatoriamente uma amostra de 55 casos para análise radiográfica, realizada por dois autores distintos (Galia C. R. e Diesel C. V.), separadamente e em momentos distintos. A concordância entre os avaliadores foi aferida pelo índice kappa.

Para a análise radiográfica, foram utilizadas radiografias de bacia em anteroposterior e avaliadas a migração axial da 


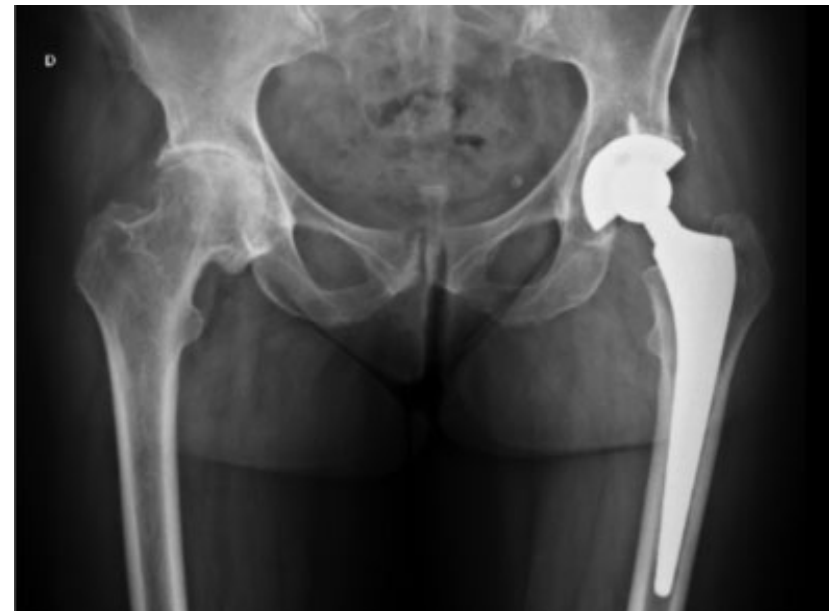

Fig. 3 Aspecto radiográfico da haste não cimentada Logical artroplastia do quadril esquerdo realizada em 2012.

haste, a presença de stress shielding, a presença de linhas de radioluscência nas zonas de Gruen e a formação de pedestal na ponta da haste. A migração axial foi considerada presente quando a distância do topo do grande trocanter-ombro da haste estivesse $>5 \mathrm{~mm}$, com um intervalo de 1 ano entre as radiografias (-Fig. $\mathbf{3}$ ).

Consideramos o afrouxamento radiográfico quando observada migração axial $>5 \mathrm{~mm}$, especialmente com progressão ao longo dos anos e associada com formação de pedestal na ponta da haste. Utilizaram-se, também, a formação de linhas de radioluscência $>2 \mathrm{~mm}$, progressivas, nas zonas de Gruen, como marcador de afrouxamento do implante.

As curvas de sobrevida foram calculadas pelo teste de Kaplan-Meier e expressas nas respectivas curvas de sobrevida. As diferenças de sobrevida entre os sexos foram avaliadas pelo teste de Mantel-Cox. As demais variáveis numéricas forram apresentadas em percentuais.

\section{Resultados}

Foram incluídos no estudo 632 pacientes, todos tendo realizado cirurgia de ATQ utilizando o implante LOGICAL CM. Destes, $54,7 \%$ (346) eram do sexo feminino e $45,3 \%$ (286) eram do sexo masculino. O seguimento médio total foi de $6,41 \pm 3,1$ anos (média \pm desvio padrão [DP]) com mínimo de 2 anos de acompanhamento e máximo de 13 anos.

Para um entendimento melhor da análise, os sujeitos da pesquisa foram divididos em 2 grupos de momentos distintos: pacientes operados de janeiro de 2004 a dezembro de 2009 (totalizando 244 pacientes) e pacientes operados de janeiro de 2010 a dezembro de 2015 (totalizando 388 pacientes).

Dos pacientes operados de janeiro de 2004 a dezembro de 2009, o seguimento médio foi de $9,73 \pm 1,42$ anos (média $\pm \mathrm{DP}$ ), com mínimo de 8 anos e máximo de 13 anos de acompanhamento. A sobrevida destes pacientes está exibida nas -Figs. 4-6.

Dos pacientes operados de janeiro de 2010 a dezembro de 2015, o seguimento médio foi de 4,32 $\pm 1,74$ anos (média \pm DP), com mínimo de 2 anos e máximo de 8 anos de acompanhamento. A sobrevida destes pacientes está exibida nas -Figs. 7-9.

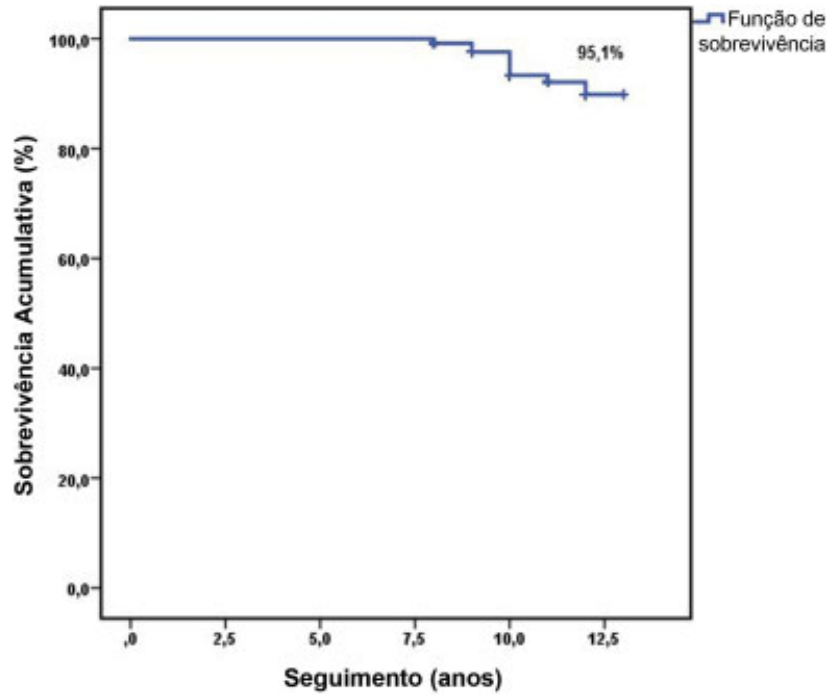

Fig. 4 Sobrevida do implante LOGICAL CM dos pacientes operados entre janeiro de 2004 e dezembro de 2009. Um total de 95,1\% dos implantes tiveram uma sobrevida média de 12,72 $\pm 0,09$ anos (média \pm desvio padrão), sendo 12,57-12,87 anos o intervalo de confiança.

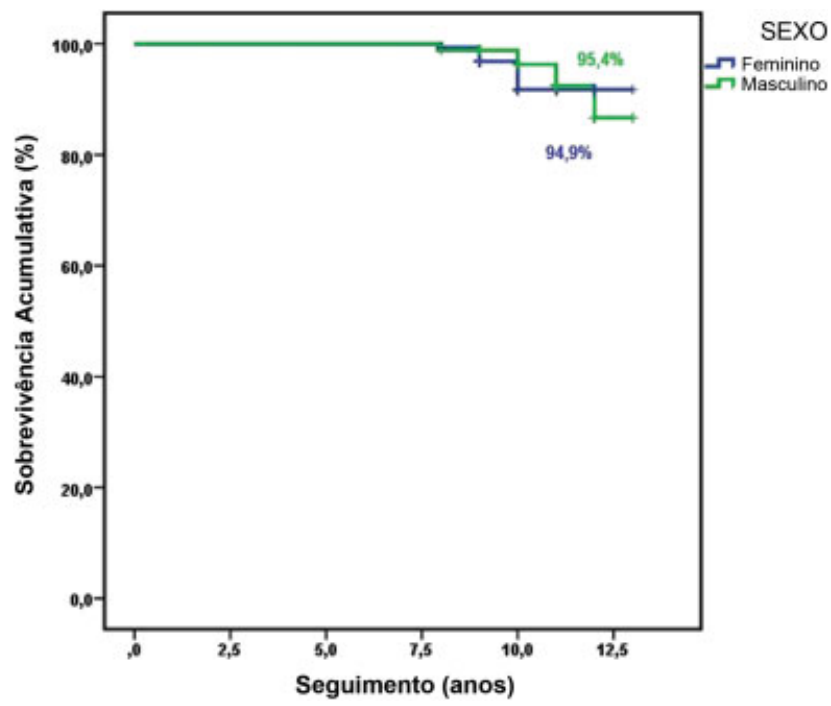

Fig. 5 Sobrevida do implante LOGICAL CM dos pacientes operados entre janeiro de 2004 e dezembro de 2009 avaliando diferenças entre os sexos. Não houve diferença na sobrevida média do implante comparando os sexos masculino e feminino. Foi utilizado teste de Log Ranl (Mantel-Cox) ( $p=0,878$ ). Sobrevida média de $12,74 \pm 0,10$ anos para o sexo feminino e de $12,73 \pm 0,13$ meses para o sexo masculino.

Os principais tamanhos dos componentes femorais utilizados estão dispostos na - Fig. 10.

Nenhum paciente foi revisado por dor residual na coxa, ou por migração axial da haste $>5 \mathrm{~mm}$. No período, foram observadas 14 fraturas periprotéticas transoperatórias (0,02\%), a maior parte resolvida por cerclagem com fios metálicos.

Não foram observados casos de atrofia óssea (stress shielding) na amostra radiográfica analisada.

\section{Discussão}

A crença norteia muitos passos da evolução do conhecimento. A fusão da crença e de princípios físicos, mecânicos 


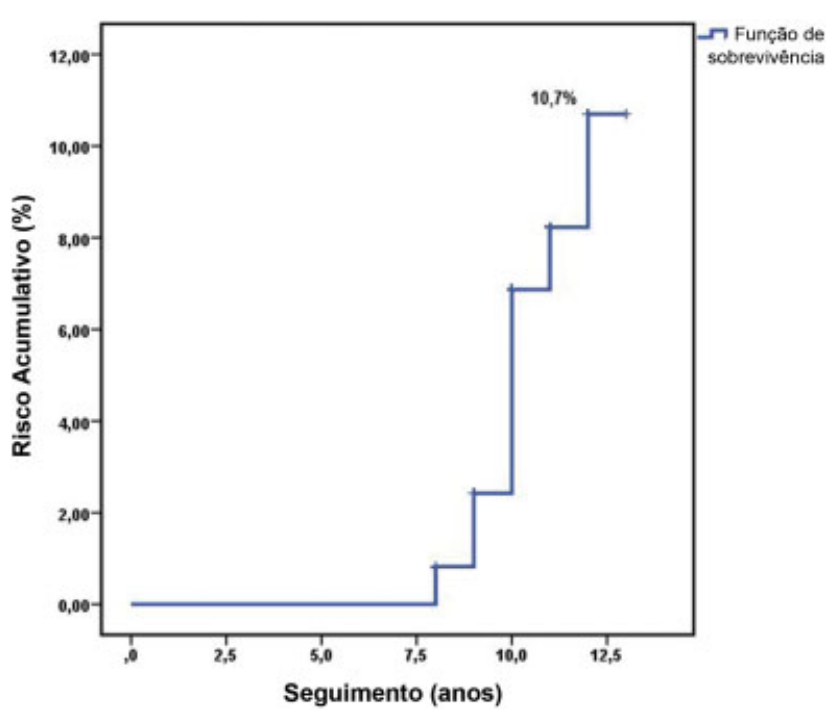

Fig. 6 Risco cumulativo de falha do implante LOGICAL CM dos pacientes operados entre janeiro de 2004 e dezembro de 2009 ao longo de 12,5 anos foi de $10,7 \%$.

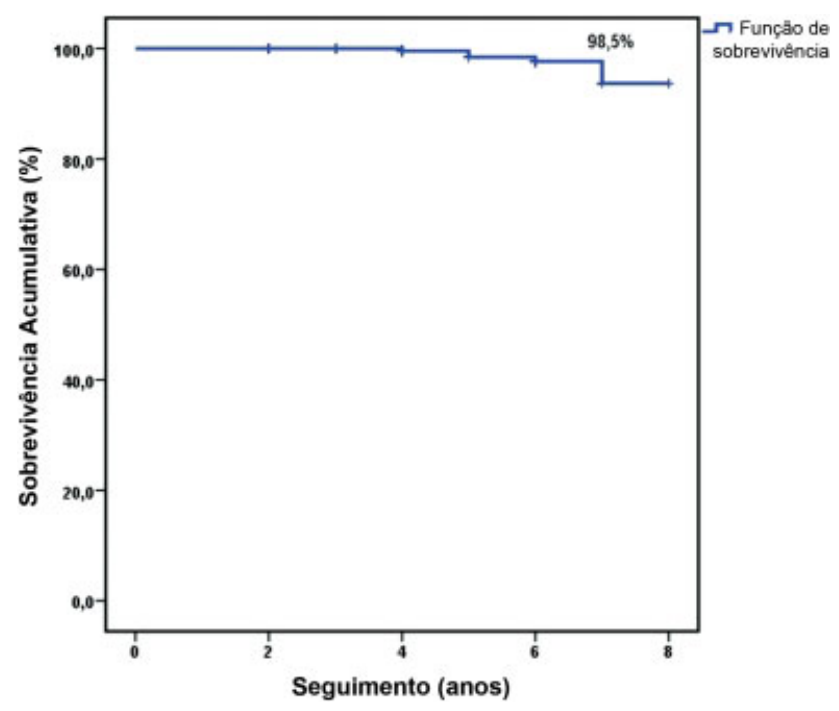

Fig. 7 Sobrevida do implante LOGICAL CM dos pacientes operados entre janeiro de 2004 e dezembro de 2009 . Um total de $98,5 \%$ dos implantes tiveram uma sobrevida média de 7,89 $\pm 0,04$ anos (média \pm desvio padrão), sendo 7,81-7,98 anos o intervalo de confiança.

e biológicos na área da ortopedia pode resultar em soluções, produtos de sucesso ou não. Institucionalmente, havia a crença de que o design mais adequado para uma haste femoral não cimentada seria o quadrangular e cuneiforme. Esta crença era sustentada pelos pilares mecânicos e biológicos já estudados por diversos autores e com sucesso comprovado ao longo dos anos como nas hastes femorais CLS (Zimmer. Warsaw, Indiana, USA). ${ }^{5-7}$ O resultado disso foi o desenvolvimento de uma haste não cimentada, quadrangular, cuneiforme, com porosidade e aletas proximais.

A haste Logical foi utilizada nas mais diversas situações e indicações, como nas ATQs, nas artroplastias parciais para o tratamento das fraturas do fêmur proximal, no tratamento das lesões metastáticas do terço proximal do fêmur e nas

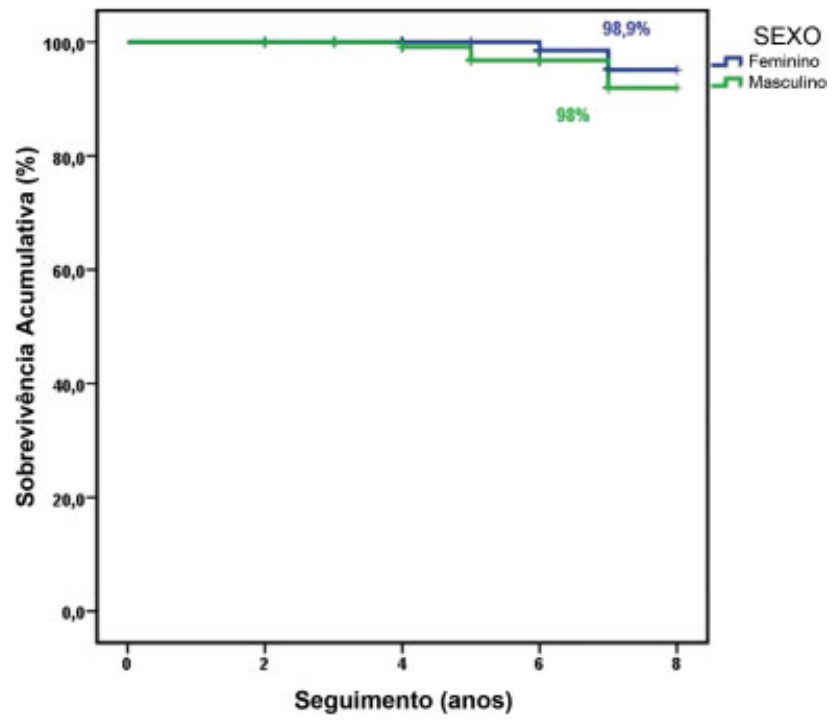

Fig. 8 Sobrevida do implante LOGICAL CM dos pacientes operados entre janeiro de 2004 e dezembro de 2009 avaliando diferenças entre os sexos. Não houve diferença na sobrevida média do implante comparando os sexos masculino e feminino. Foi utilizado teste de Log Ranl (Mantel-Cox) $(p=0,287)$. Sobrevida média de 7,94 $\pm 0,04$ anos para o sexo feminino e de $7,85 \pm 0,06$ anos para o sexo masculino.

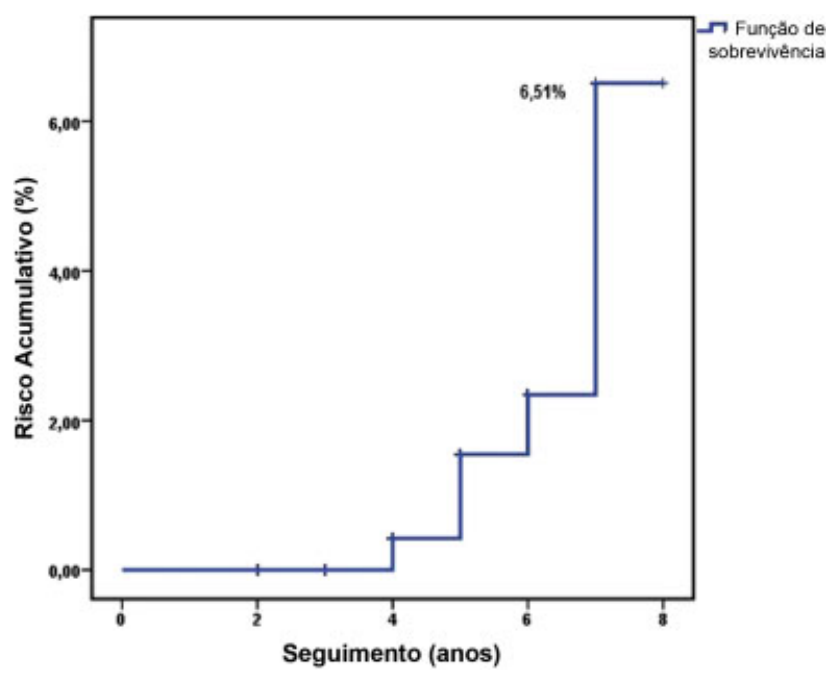

Fig. 9 O risco cumulativo de falha do implante LOGICAL CM dos pacientes operados entre janeiro de 2004 e dezembro de 2009 ao longo de 8 anos foi de $6,51 \%$.

revisões do componente femoral. Foram implantadas, desde o ano de 2004, mais de 1.000 hastes femorais em nossa instituição.

Os anos iniciais foram dedicados aos ajustes e aperfeiçoamentos nos instrumentais, além da observação da evolução pós-operatória dos pacientes. Também foram os anos despendidos para curva de aprendizado do implante.

A observação da evolução dos primeiros casos foi encorajadora, e passou-se a uma maior liberalidade nas indicações dessa haste não cimentada. A faixa etária, os conceitos de Dorr e do índice morfocortical foram, aos poucos, deixando de ser critérios utilizados na indicação desta haste não cimentada. 


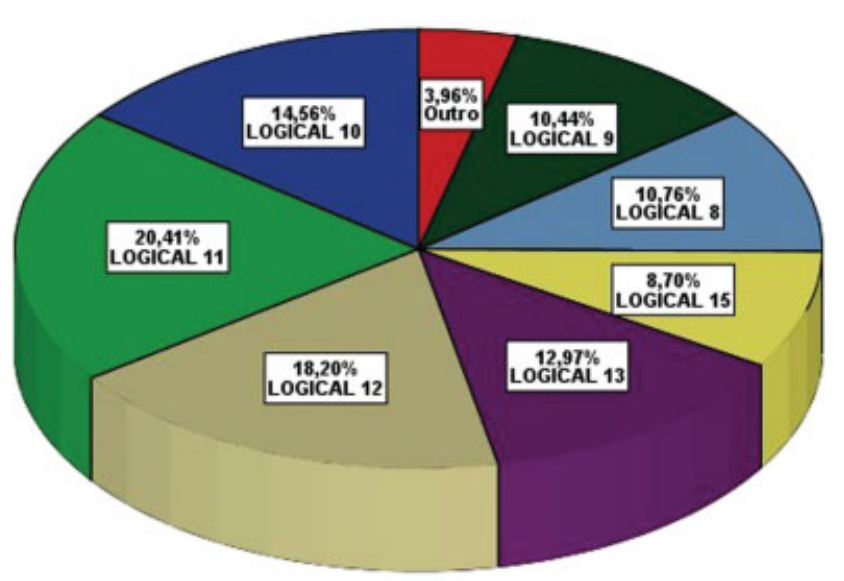

Fig. 10 Tamanhos dos componentes femorais.

Não encontramos, na amostra radiográfica avaliada, migração axial da haste femoral. Embora a totalidade das radiografias não tenha sido analisada, o design do implante e sua adaptação ao canal femoral, além do baixo número de indicação de revisões, nos faz crer que este evento seja realmente raro.

Observamos um total de 14 fraturas periprotéticas transoperatórias (0.02\%). Embora os números encontrados na literatura sejam variáveis (entre $0,1 \%$ e $27,8 \%$ ), ${ }^{8}$ atribuímos este baixo número de casos à experiência institucional com esta haste não cimentada - a única utilizada na instituição desde 2004.

Também não encontramos, como não tínhamos a expectativa de identificar, na amostra radiográfica analisada, indícios de atrofia óssea cortical (stress shielding). Atribuímos isso às características deste implante não cimentado (em dupla cunha, com porosidade proximal e biocompatível). Estas características somadas, entre outras, aumentam a distribuição de carga na interface implante-osso e reduzem a incidência da atrofia óssea.

Embora um seguimento de médio prazo ainda seja insuficiente em termos de seguimento de implantes ortopédicos, os resultados obtidos e expressos nas curvas de sobrevida são muito animadores.
Atualmente, já estão adaptados à haste Logical, as cabeças de cerâmica e seu conjunto acetabular conta com o polietileno tipo crosslinked. Nos casos analisados, esperamos um maior número de indicação de revisão destas hastes ao longo do tempo, visto o par tribológico utilizado, na grande maioria deles, ser o metal-polietileno de ultraelevado peso molecular. Aspectos relacionados com a geração de partículas e sua repercussão na sobrevida das artroplastias são bem conhecidos. Consideramos, dessa forma, os próximos anos como muito desafiadores.

Declaração de Conflito de Interesses

Um dos autores, Dr. Macedo C. A. S., declara haver conflito de interesses, visto ser o responsável pelo desenvolvimento da haste femoral Logical e ter cedido a tecnologia à empresa Baumer, Mogi Mirim, SP, Brasil.

\section{Referências}

1 Aldinger PR, Jung AW, Breusch SJ, Ewerbeck V, Parsch D. Survival of the cementless Spotorno stem in the second decade. Clin Orthop Relat Res 2009;467(09):2297-2304

2 Johanson PE, Antonsson M, Shareghi B, Kärrholm J. Early Subsidence Predicts Failure of a cemented femoral stem with minor design changes. Clin Orthop Relat Res 2016;474(10):2221-2229

3 Kitamura S, Hasegawa Y, Iwasada S, Yamauchi K, Kawamoto K, Kanamono T, et al. Catastrophic failure of cementless total hip arthroplasty using a femoral component without surface coating. J Arthroplasty 1999;14(08):918-924

4 Macedo CAS. Desenvolvimento de haste femoral não cimentada nacional, validada por normas internacionais [tese]. Porto Alegre: Universidade Federal do Rio Grande do Sul; 2007

5 Rozkydal Z, Janícek P, Havlícek V, Pazourek L. [Long-term results of use of the CLS stem in primary total hip arthroplasty]. Acta Chir Orthop Traumatol Cech 2009;76(04):281-287

6 Evola FR, Evola G, Graceffa A, Sessa A, Pavone V, Costarella L, et al. Performance of the CLS Spotorno uncemented stem in the third decade after implantation. Bone Joint J 2014;96-B(04):455-461

7 Yamasaki T, Yasunaga Y, Mori R, Hamanishi M, Shoji T, Ochi M. The Cementless Spotorno stem in THA: 10 year results. Hip Int 2014; 24(01):98-102

8 Sidler-Maier CC, Waddell JP. Incidence and predisposing factors of periprosthetic proximal femoral fractures: a literature review. Int Orthop 2015;39(09):1673-1682 